\title{
Imprecise linear filtering: a second step
}

\author{
Olivier Strauss ${ }^{1}$ Agnès Rico ${ }^{1,2}$ \\ ${ }^{1}$ LIRMM Université Montpellier II, 61 rue Ada, 34392 Montpellier cedex 5, France \\ ${ }^{2}$ ERIC Université Claude Bernard Lyon 1, 43 bld du 11 novembre 191869622 Villeurbanne, France
}

\begin{abstract}
Linear digital signal processing consists in convoluting the input sampled signal with the discrete version of the impulse response of a filter designed by an expert. More than often, a unique impulse response does not represent the complete knowledge of the expert who should have proposed more than one appropriate filter. In a recent paper, we have proposed an extension of the finite impulse response filtering that able to represent the fact that the filter is imprecisely known. This extension leads to compute an interval-valued filtered signal. In this paper, we propose a natural follow-up of this work by considering interval-valued input signals and replacing the Choquet integral by the Šipoš integral.
\end{abstract}

Keywords: Linear filtering, interval-valued signal, Choquet and Šipoš integrals, capacities, imprecise knowledge.

\section{Introduction}

In signal processing, filtering consists of modifying a real input signal by blocking pre-specified particular components (usually frequency components or random components). By contrast with an analog filter, which directly operates on a continuous signal, a digital filter operates on digital samples and performs a mathematical manipulation that results in output samples having theoretically pre-required properties. Since the digital samples to be processed are usually obtained by sampling a continuous signal and since the output samples are generally converted in a continuous output signal via a D/A converter, most digital filters are designed to mimic analog filters. More precisely, the prerequired properties of the output digital signal are specified in the continuous domain.

Finite impulse response (FIR) filtering is one of the most popular method due to the low complexity of the algorithms it involves and the high simplicity of the representation it carries on. The mathematical manipulation involved in FIR filtering consists of convolving the input samples with a particular digital signal called the impulse response of the filter. The ability of the mathematical manipulation to achieve the desired filtering is strongly linked with the ability of the user to specify the appropriate impulse response of the filter. Despite the expertise most of users have developed, this specification still represents a difficulty. To answer to this difficulty, a skilled practitioner usually defines an objective criterion (e.g. a distance between a desired output and the filtered output in different situations) and selects the FIR that optimizes this predefined criterion. However, the objectivity of the criterion is still debatable. In fact, slightly changing the criterion (e.g. moving from a $L_{2}$ to a $L_{1}$ distance) more often than not leads to a strong change in the selected FIR. Another appropriate answer to the difficulty of defining a specific FIR is defining a set of possible appropriate impulse response. Such a filtering algorithm would naturally lead to a set of filtered outputs. Replacing a single FIR by a set of FIR can lead, as a drawback, to a drastic increase of the the filtering algorithm's complexity, particularly if the cardinality of this set is infinite (e.g. when representing the set of all low-pass Butterworth filters having a cutoff frequency that belongs to an interval $\left.\left[f_{\min }, f_{\max }\right]\right)$. In a recent article [?], we have proposed a set-valued filtering method having an algorithmic complexity which is comparable to the complexity of usual filtering algorithms. The method we propose is based on representing a convex set of FIR by a convex capacity and extending the convolution operation by the use of the Choquet integral. Our algorithm computes the upper and lower bounds of an interval-valued filtered output which is the convex set of all the values that would have been obtained by using the conventional approach with all the FIR belonging to the considered set. Moreover, as shown in [?], the imprecision of the output can be used as a marker of the random noise of the input signal, or more precisely, on the influence of this noise on the statistical variations in the output.

In [?], the approach we propose is based on the asymmetric Choquet integral. It sometimes leads to interval-valued output that are not specific enough for some applications e.g. automatic control. In this article, we propose two extensions of this work. First, we propose to generalize these filtering method to interval-valued inputs. This extension aims at accounting for a known imprecision due to error-calibration of the measurement process, or for a known statistical error by means of confidence intervals. The signal to be filtered can also be interval-valued because it is the output of a previous interval-valued filtering process. Second, we propose to consider the symmetric Choquet integral (or Šipoš integral) instead of the asymmetric Choquet integral. This replacement leads to more specific 
interval-valued outputs. Moreover, we show that the interval-valued output given by the symmetric approach is always symmetrically distributed in the the interval-valued output given by the asymmetric approach.

This article is organized as follows. Section ?? presents the framework and notations. Section ?? deals with symmetric and asymmetric Choquet integrals. Section ?? recalls some basis on Minkowski additive and subtractive operations for intervals. Section ?? proposes to extend imprecise expectation operators proposed in [?] to intervals and introduces a new expectation operator based on the Šipoš integral. Section ?? presents some experiments to illustrate most of the properties mentioned in this paper.

\section{Framework and notations}

Let $X=\left(X_{n}\right)_{n=1, \ldots, N}$ be a sequence of $N$ digital samples of a signal. The set $\{1, \cdots, N\}$ will be denoted $\Omega$. Hence $X$ can be viewed as a real function on a finite set $\Omega$. The set of these functions is denoted $V$. Let $\rho=\left(\rho_{i}\right)_{i \in \mathbb{Z}}$ be the finite impulse response of the considered filter ${ }^{1}$. The computation of $Y_{k}$, the $k$-th component of the filter output, is given by $Y_{k}=\sum_{n=1}^{N} \rho_{k-n} X_{n}$. When the impulse response is positive and has a unitary gain $\left(\forall i \in \mathbb{Z}, \rho_{i} \geq 0\right.$ and $\left.\sum_{i \in \mathbb{Z}} \rho_{i}=1\right)$, it can be considered as a probability mass function inducing a probability measure $P$ on each subset $A$ of $\mathbb{Z}$ by $P(A)=$ $\sum_{i \in A} \rho_{i}$. This special type of impulse responses are often called summative kernels [?], or simply kernels, when used to ensure interplay between continuous and discrete domains. Thus, computing $Y_{k}$ is equivalent to computing a discrete expectation operator involving a probability measure $P_{k}$ induced by $\left(\rho_{k-n}\right)_{n \in \mathbb{Z}}$, the probability distribution obtained by translating the probability distribution $\rho$ over $k$ : $Y_{k}=\sum_{n=1}^{N} \rho_{k-n} X_{n}=E_{P_{k}}(X)$. According to this interpretation, the probability $P_{k}$ defines a probabilistic neighborhood of the $k$-th sample. As the impulse response is finite, it has a bounded support, i.e. $\exists N \in \mathbb{N}, i \notin[-N, N] \Rightarrow \rho_{i}=0$. This bounded support will be referred to throughout this paper as the radius of the summative kernel.

The approach we propose in [?] is to compute an interval-valued signal containing all outputs of filtering process involving a coherent family of conventional linear filters. This approach is based on an extension of the expectation operator involving an asymmetric Choquet integral.

\section{Symmetric and asymmetric Choquet integral}

A capacity $v$ is a set function $v: \mathcal{P}(\Omega) \rightarrow[0,1]$ such that $v(\varnothing)=0(\varnothing$ is the empty set of

\footnotetext{
${ }^{1}$ The impulse response of the filter being finite, $\exists K \in \mathbb{Z}$ such that $\forall i \geq K, \rho_{i}=0$. The general formulation is easier to be manipulated.
}

$\Omega), v(\Omega)=1$, and $\forall A \subseteq B \Rightarrow v(A) \leq v(B)$. $v^{c}$, the conjugate capacity of $v$, is a capacity defined by $\forall A \in \mathcal{P}(\Omega), v^{c}(A)=1-v\left(A^{c}\right)$ where $A^{c}$ denotes the complementary set of $A$ in $\Omega$. A capacity $v$ is concave if and only if $\forall A, B \in \mathcal{P}(\Omega), v(A \cup B)+v(A \cap B) \leq v(A)+v(B)$. The core of a concave capacity $v$ is $\operatorname{core}(v)=$ $\{P$ probalility on $\mathcal{P}(\Omega)$ such that $P(A) \leq$ $v(A), \forall A \in \mathcal{P}(\Omega)\}$.

Let $X=\left(X_{n}\right)_{n=1, \ldots, N}$ be a real function on $\Omega$. (.) denotes the permutation function on $\Omega$ such that $X$ is a non decreasing function, i.e. $X_{(1)} \leq \ldots \leq X_{(N)}$.

If $X$ is a positive real function then the Choquet integral of $X$ with respect to the capacity $v$ is:

$$
C_{v}(X)=\sum_{n=1}^{N} X_{(n)}\left(v\left(A_{(n)}\right)-v\left(A_{(n+1)}\right)\right),
$$

where $\forall i \in \Omega, A_{(i)}$ is the subset of $\Omega$ defined by $A_{(i)}=\{(i), \ldots,(N)\}$ and $A_{(N+1)}=\varnothing$.

If $X$ is a real function then there exists two possibilities for extending the Choquet integral: the asymmetric Choquet integral, also called Choquet integral, and the symmetric Choquet integral, also called Šipoš integral.

Let $r$ be the index such that

$X_{(1)} \leq \ldots \leq X_{(r)}<0 \leq X_{(r+1)} \leq \ldots \leq$ $X_{(N)}$. Hence two positive fonctions can be defined. $X^{-}$is the function defined by $\forall n \in \Omega, X_{n}^{-}=$ $\max \left(-X_{n}, 0\right)$ and $X^{+}$is the function defined by $\forall n \in \Omega, X_{n}^{+}=\max \left(X_{n}, 0\right)$. Thus, $\forall n \in \Omega$, $X_{n}=X_{n}^{+}-X_{n}^{-}$.

The asymmetric Choquet integral of the function $X$ with respect to a capacity $v$ is:

$\check{C}_{v}(X)=C_{v}\left(X^{+}\right)-C_{v^{c}}\left(X^{-}\right)$.

The Šipoš integral of the function $X$ with respect to a capacity $v$ is:

$$
\check{S}_{v}(X)=C_{v}\left(X^{+}\right)-C_{v}\left(X^{-}\right) \text {. }
$$

An important property of these asymmetric and symmetric Choquet integrals will be used in the sequel: $\check{C}_{v}(X)$ and $\breve{S}_{v}(X)$ are monotonic with respect to $X$.

\section{Minkowski additive operations for real intervals and interval-valued vectors}

\subsection{Real intervals}

The real intervals are denoted $[x]=[\underline{x}, \bar{x}]$ where $\underline{x}$ (rsp. $\bar{x}$ ) is the lower (rsp. upper) bound of the interval $[x]$. The set of all real intervals is denoted IIR.

- The Minkowski addition of two intervals $[x]$ and $[y]$ is $[x] \oplus[y]=[\underline{x}+\underline{y}, \bar{x}+\bar{y}]$.

- The dual Minkowski addition of two intervals $[x]$ and $[y]$ is $[x] \boxplus[y]=[\min (\underline{x}+\bar{y}, \bar{x}+$ $\underline{y}), \max (\underline{x}+\bar{y}, \bar{x}+\underline{y})]$.

If $0 \in[y]$, then the Minkowski addition can be interpreted as a dilatation and the dual Minkowski addition can be interpreted as an erosion [?]. 
- The Minkowski subtraction of two intervals $[x]$ and $[y]$ is $[x] \ominus[y]=[\underline{x}, \bar{x}] \oplus[-\bar{y},-\underline{y}]=[\underline{x}-$ $\bar{y}, \bar{x}-y]$.

- The dual Minkowski subtraction of two intervals $[x]$ and $[y]$ is $[x] \boxminus[y]=[\underline{x}, \bar{x}] \boxplus[-\bar{y},-\underline{y}]=$ $[\min (\underline{x}-\underline{y}, \bar{x}-\bar{y}), \max (\underline{x}-\underline{y}, \bar{x}-\bar{y})]$.

Note that the $\boxminus$ operator is identical to the difference operator defined by Hukahara in [?] when $[a]=[b] \oplus[x]$ has a solution.

\subsection{Interval-valued vectors}

In this paper, we consider interval-valued discrete functions, which can be represented by intervalvalued vectors $[\mathbf{X}]$ :

$$
[\mathbf{X}]=\left(\left[\underline{x_{1}}, \overline{x_{1}}\right], \quad \cdots,\left[\underline{x_{n}}, \overline{x_{n}}\right]\right)^{t}
$$

where $t$ is the transposition function on vectors. The set of interval-valued vectors is denoted II $V$.

Considering the interval-valued vector $[\mathbf{X}] \in \mathbb{I} V$, the two following real vectors can be defined: $\underline{X}=\left(\underline{x_{1}}, \cdots, \underline{x_{n}}\right)^{t}$ and $\bar{X}=\left(\overline{x_{1}}, \cdots, \overline{x_{n}}\right)^{t}$.

Based on these two vectors, we extend the notation $[\underline{x}, \bar{x}]$ to vectors by denoting: $[\mathbf{X}]=[\underline{X}, \bar{X}]=$ $\{Y \in V \mid \underline{X} \leq Y \leq \bar{X}\}$ where $X \leq Y$ if and only if $X_{n} \leq Y_{n} \forall n \in\{1, \ldots, N\}$. We also extend the Minkowski operators to vectors by denoting: $\forall[\mathbf{X}],[\mathbf{Y}] \in \mathbb{I} V,[\mathbf{Z}]=[\mathbf{X}] \diamond[\mathbf{Y}]$ the intervalvalued vector such that $\forall n \in \Omega,\left[z_{n}\right]=\left[x_{n}\right] \diamond\left[y_{n}\right]$ with $\diamond \in\{\oplus, \boxplus, \ominus, \boxminus\}$.

\section{Imprecise expectation operators}

As remarked in Section ??, filtering a real signal can be seen as an expectation operation involving a probability measure based on the impulse response of the filter. Since the core of a concave capacity is a convex set of probabilities, a capacity allows an imprecise representation of a probability measure, i.e. an ill-known impulse response. In this section, we define two imprecise expectation operators $\underline{\bar{E}}$ and $\underline{\underline{B}}$ that extend the usual expectation E operator to concave capacities. Those two imprecise expectation operators have an imprecise output representing the sets of output that should have been obtained by using an expectation operator based on a probability measure belonging to the considered convex set. These two outputs correspond to two different filtering contexts.

We first achieve these extensions to real valued discrete functions. We then generalize these extensions to interval-valued discrete functions.

Let us first recall a classical result proved by D. Denneberg [?]:

Proposition 1 If $v$ is a concave capacity then

- $\forall X \in V, \check{C}_{v}(X)=\sup _{P \in \operatorname{core}(v)} \mathrm{E}_{P}(X)$,

- $\forall X \in V, \check{C}_{v^{c}}(X)=\inf _{P \in \operatorname{core}(v)} \mathrm{E}_{P}(X)$,
- $\forall X \in V, \check{C}_{v^{c}}(X) \leq \check{C}_{v}(X)$,

where $\mathrm{E}_{P}(X)$ it the usual expectation of the function $X$ based on the probability measure $P$.

\subsection{Interval-valued expectation operators for real functions}

According to Proposition ??, for any function $X \in$ $V$ the interval $\left[\check{C}_{v^{c}}(X), \check{C}_{v}(X)\right]$ always exists while either $\left[\check{S}_{v^{c}}(X), \check{S}_{v}(X)\right]$ or $\left[\check{S}_{v}(X), \check{S}_{v^{c}}(X)\right]$ exists.

The extension $\underline{\underline{E}}$ is based on the Choquet integral while the extension $\underline{\underline{\underline{G}}}$ is based on the Šipoš integral.

\section{Definition 1}

$$
\begin{aligned}
& \overline{\underline{\mathrm{E}}}_{v}(X)=\left[\check{C}_{v^{c}}(X), \check{C}_{v}(X)\right] \\
& \underline{\overline{\mathrm{B}}}_{v}(X)=\left[\min \left(\check{S}_{v^{c}}(X), \check{S}_{v}(X)\right), \max \left(\check{S}_{v^{c}}(X), \check{S}_{v}(X)\right)\right] .
\end{aligned}
$$

The properties of operator $\underline{\mathrm{E}}_{v}$ are presented in [?]. We point out a certain number of these properties here. Let $v$ be a concave capacity. If, for all probabilities $P \in \operatorname{core}(v)$, all the expectations $\mathrm{E}_{P}(X)$ are equal, then $\check{C}_{v^{c}}(X)=\check{C}_{v}(X)$. If $X$ is a function equal to a constant value $c$ then $\check{C}_{v^{c}}(X)=\check{C}_{v}(X)=c$.

As shown in [?] the following result holds: $\overline{\mathrm{E}}_{v}(X)=\underline{\mathrm{E}}_{v}\left(X^{+}\right) \ominus \underline{\mathrm{E}}_{v}\left(X^{-}\right)$.

Finally, by construction, if $X \in V$ is a positive function, then the asymmetric Choquet integral and the Šipoš integral coincide with the Choquet integral, and thus $\overline{\mathrm{E}}_{v}(X)=\underline{\underline{\mathrm{B}}}_{v}(X)$.

\section{Proposition 2}

$$
\forall X \in V, \overline{\underline{\underline{G}}}_{v}(X) \subseteq \overline{\mathrm{E}}_{v}(X) .
$$

Proof: $\forall X \in V, C_{v^{c}}(X) \leq C_{v}(X)$, so using the functions $X^{+}$and $X^{-}$we have

$$
\check{S}_{v}(X)=C_{v}\left(X^{+}\right)-C_{v}\left(X^{-}\right) \leq C_{v}\left(X^{+}\right)-
$$
$C_{v^{c}}\left(X^{-}\right)=\check{C}_{v}(X)$,

$\check{S}_{v^{c}}(X)=C_{v^{c}}\left(X^{+}\right)-C_{v^{c}}\left(X^{-}\right) \leq C_{v}\left(X^{+}\right)-$ $C_{v}\left(X^{-}\right)=\check{C}_{v}(X)$

$\breve{S}_{v^{c}}(X)=C_{v^{c}}\left(X^{+}\right)-C_{v^{c}}\left(X^{-}\right) \geq C_{v^{c}}\left(X^{+}\right)-$ $C_{v}\left(X^{-}\right)=\check{C}_{v^{c}}(X)$,

$\check{C}_{v^{c}}(X)-\check{S}_{v}(X)=C_{v^{c}}\left(X^{+}\right)-C_{v}\left(X^{-}\right)-$ $C_{v}\left(X^{+}\right)+C_{v}\left(X^{-}\right)=C_{v^{c}}\left(X^{+}\right)-C_{v}\left(X^{+}\right) \leq 0$.

So we have proved that $\check{C}_{v_{\breve{c}}^{c}}(X) \leq$ $\min \left(\check{S}_{v}(X), \check{S}_{v^{c}}(X)\right)$ and $\max \left(\check{S}_{v}(X), \check{S}_{v^{c}}(X)\right) \leq$ $\check{C}_{v}(X)$.

Proposition $3 \forall X \in V$, the inclusion $\overline{\underline{\underline{G}}}_{v}(X) \subseteq$ $\overline{\mathrm{E}}_{v}(X)$ is symmetric i.e. $\check{C}_{v}(X)-\bar{S}(X)=\underline{S}(X)-$ $\check{C}_{v^{c}}(X)$.

Proof: The inclusion is symmetric if and only if $\check{C}_{v}(X)-\bar{S}=\underline{S}-\check{C}_{v^{c}}(X)$ and $\check{C}_{v}(X)-\underline{S}=\bar{S}-$ $\check{C}_{v^{c}}(X)$.

Let $v$ be a capacity,

$$
\begin{aligned}
& \check{C}_{v}(X)=C_{v}\left(X^{+}\right)-C_{v^{c}}\left(X^{-}\right) \\
& =C_{v}\left(X^{+}\right)-C_{v}\left(X^{-}\right)+C_{v}\left(X^{-}\right)-C_{v^{c}}\left(X^{-}\right) \\
& =\check{S}_{v}(X)+\left(C_{v}\left(X^{-}\right)-C_{v^{c}}\left(X^{-}\right)\right) .
\end{aligned}
$$


Note that if we consider $v^{c}$ then $\check{C}_{v^{c}}(X)=\check{S}_{v^{c}}(X)-$ $\left(C_{v}\left(X^{-}\right)-C_{v^{c}}\left(X^{-}\right)\right)$.

So we have $\check{C}_{v}(X)-\check{S}_{v}(X)=\check{S}_{v^{c}}(X)-\check{C}_{v^{c}}(X)$.

$$
\begin{aligned}
& \check{C}_{v}(X)=C_{v}\left(X^{+}\right)-C_{v^{c}}\left(X^{-}\right) \\
& =C_{v}\left(X^{+}\right)-C_{v^{c}}\left(X^{+}\right)+C_{v^{c}}\left(X^{+}\right)-C_{v^{c}}\left(X^{-}\right) \\
& =\check{S}_{v^{c}}(X)+\left(C_{v}\left(X^{+}\right)-C_{v^{c}}\left(X^{+}\right)\right) \\
& \check{C}_{v^{c}}(X)=C_{v^{c}}\left(X^{+}\right)-C_{v}\left(X^{-}\right) \\
& =C_{v}\left(X^{+}\right)-C_{v}\left(X^{+}\right)+C_{v^{c}}\left(X^{+}\right)-C_{v}\left(X^{-}\right) \\
& =\check{S}_{v}(X)-\left(C_{v}\left(X^{+}\right)-C_{v^{c}}\left(X^{+}\right)\right)
\end{aligned}
$$

Therefore $\check{C}_{v}(X)-\check{S}_{v^{c}}(X)=\check{S}_{v}(X)-\check{C}_{v^{c}}(X)$.

The previous proposition can be summarized in the following figure:

$$
\begin{aligned}
& \left(C_{v}-C_{v^{c}}\right)\left(X^{-}\right) \quad\left(C_{v}-C_{v^{c}}\right)\left(X^{+}\right)
\end{aligned}
$$

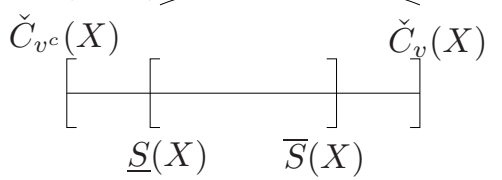

$$
\begin{aligned}
& \left(C_{v}-C_{v^{c}}\right)\left(X^{+}\right) \quad\left(C_{v}-C_{v^{c}}\right)\left(X^{-}\right)
\end{aligned}
$$

with $\underline{S}(X)=\min \left(\check{S}_{v^{c}}(X), \check{S}_{v}(X)\right)$ and $\bar{S}(X)=\max \left(\check{S}_{v^{c}}(X), \check{S}_{v}(X)\right)$.

A straightforward corollary of this proposition is that $\underline{\underline{\underline{G}}}_{v}(X)$ and $\underline{\mathrm{E}}_{v}(X)$ have the same middle value.

Proposition $4\left(C_{v}-C_{v^{c}}\right)\left(X^{-}\right)=\left(C_{v}-C_{v^{c}}\right)\left(X^{+}\right)$ if and only if $\underline{S}=S_{v^{c}}(X)=\bar{S}=S_{v}(X)$.

\section{Proof:}

$$
\begin{aligned}
& S_{v^{c}}(X)-S_{v}(X)= \\
& C_{v^{c}}\left(X^{+}\right)-C_{v^{c}}\left(X^{-}\right)-\left[C_{v}\left(X^{+}\right)-C_{v}\left(X^{-}\right)\right]= \\
& \left(C_{v^{c}}-C_{v}\right)\left(X^{+}\right)-\left(C_{v^{c}}-C_{v}\right)\left(X^{-}\right) .
\end{aligned}
$$

For $X \in V$, if the interval $\underline{\underline{G}}_{v}(X)$ is reduced to a real value, then this value is the center of the interval $\underline{\mathrm{E}}_{v}(X)$.

\section{Proposition 5}

$$
\underline{\underline{\underline{B}}}_{v}(X)=\underline{\mathrm{E}}_{v}\left(X^{+}\right) \boxminus \underline{\mathrm{E}}_{v}\left(X^{-}\right) \text {. }
$$

Proof: We have $\overline{\mathrm{E}}_{v}\left(X^{+}\right)=\left[\check{C}_{v^{c}}\left(X^{+}\right), \check{C}_{v}\left(X^{+}\right)\right]$ and $\overline{\mathrm{E}}_{v}\left(X^{-}\right)=\left[\check{C}_{v^{c}}\left(X^{-}\right), \check{C}_{v}\left(X^{-}\right)\right]$. Hence using the definition of $\boxminus$, the lower bound of $\overline{\mathrm{E}}_{v}\left(X^{+}\right) \boxminus$ $\overline{\mathrm{E}}_{v}\left(X^{-}\right)$is $\min \left(\check{C}_{v^{c}}\left(X^{+}\right)-\check{C}_{v^{c}}\left(X^{-}\right), \check{C}_{v}\left(X^{+}\right)-\right.$ $\left.\breve{C}_{v}\left(X^{-}\right)\right)$and its upper bound is $\max \left(\check{C}_{v^{c}}\left(X^{+}\right)-\right.$ $\left.\check{C}_{v^{c}}\left(X^{-}\right), \check{C}_{v}\left(X^{+}\right)-\check{C}_{v}\left(X^{-}\right)\right)$. Thus, $\overline{\mathrm{E}}_{v}\left(X^{+}\right) \boxminus$ $\overline{\mathrm{E}}_{v}\left(X^{-}\right)=\underline{\underline{\underline{G}}}_{v}(X)$.

Therefore, due to the definition of the dual Minkovski subtraction, $[z]=\overline{\underline{G}}_{v}(X)$ is the solution of either $\overline{\mathrm{E}}_{v}\left(X^{+}\right) \oplus[z]=\underline{\mathrm{E}}_{v}\left(X^{-}\right)$or $\underline{\mathrm{E}}_{v}\left(X^{-}\right) \oplus[z]=$ $\underline{\mathrm{E}}_{v}\left(X^{+}\right)$, i.e. 画 $(X)$ is the quantity that should be added to the interval-valued expectation of the positive (negative) part of the function to obtain the interval-valued expectation of the negative (positve) part of the function.

\subsection{Interval-valued expectation operators for interval-valued functions}

Let $[\mathbf{X}]=[\underline{X}, \bar{X}] \in \mathbb{I} V$ be an interval-valued vector. We propose a three steps construction of the interval-valued expectations $\underline{\mathrm{E}}_{v}([\mathbf{X}])$ and $\underline{\underline{\underline{G}}}_{v}([\mathbf{X}])$ in order to be coherent with the imprecise representation of both the function and the impulse response of the considered filter.

The first step of this construction consists in generalizing to interval-valued vectors the definition of the functions $X^{+}$and $X^{-}$.

As a preliminary remark, note that, for all $X \in$ $[\mathbf{X}]$, there exists a decomposition $X=X^{+}-X^{-}$. Considering the fact that $X \in[\mathbf{X}]$ entails $\underline{X} \leq X \leq$ $\bar{X}, \underline{X}^{+}=\underline{X} \vee 0$ is the lower bound of $X^{+}$and $\bar{X}^{+}=$ $\bar{X} \vee 0$ is its upper bound, $\vee$ being the maximum computed coordinate by coordinate, and 0 being the function that equals 0 for all $i \in \Omega$. Similarly when considering $X^{-}, X^{-} \geq \underline{X}^{-}=(-\bar{X}) \vee 0$, and $X^{-} \leq$ $\bar{X}^{-}=(-\underline{X}) \vee 0$. To summarize, $\forall X \in[\mathbf{X}], \underline{X}^{+} \leq$ $X^{+} \leq \bar{X}^{+}$and $\underline{X}^{-} \leq X^{-} \leq \bar{X}^{-}$.

The generalization we propose is the natural interval-based extension of this definition.

Definition 2 If $[\mathbf{X}]$ is an interval-valued vector, then the interval-valued vectors $\left[\mathbf{X}^{-}\right]$and $\left[\mathbf{X}^{+}\right]$are:

- $\left[\mathbf{X}^{-}\right]=\max (-[\mathbf{X}],[\mathbf{0}])$

- $\left[\mathbf{X}^{+}\right]=\max ([\mathbf{X}],[\mathbf{0}])$

where $\max (-[\mathbf{X}],[\mathbf{0}])=\{(-Y) \vee 0 \mid Y \in[\mathbf{X}]\}$ and $\max ([\mathbf{X}],[\mathbf{0}])=\{Y \vee 0 \mid Y \in[\mathbf{X}]\}$.

According to the previous definition, $\left[\mathbf{X}^{+}\right]=$ $\left[\underline{X}^{+}, \bar{X}^{+}\right],\left[\mathbf{X}^{-}\right]=\left[\underline{X}^{-}, \bar{X}^{-}\right]$and using the definition of the Minkowski subtraction we have the following relation.

\section{Proposition 6}

$$
[\mathbf{X}]=\left[\mathbf{X}^{+}\right] \ominus\left[\mathbf{X}^{-}\right] .
$$

The second step of the construction needs the extension of the operator $\underline{\bar{E}}$ to positive interval-valued vectors.

Definition 3 Let $[\mathbf{X}]=[\underline{X}, \bar{X}]$. $\in \mathbb{I} V$ be a positive interval-valued vector (i.e. $\max ([\mathbf{X}], 0)=[\mathbf{X}]$ ), the interval-valued expectation of $[\mathbf{X}]$ with respect to the capacity $v$ is:

$$
\underline{\mathrm{E}}_{v}([\mathbf{X}])=\left[C_{v^{c}}(\underline{X}), C_{v}(\bar{X})\right]
$$

\section{Proposition 7}

$\forall X \in[\mathbf{X}], \underline{\overline{\mathrm{E}}}_{v}(X) \subseteq \underline{\mathrm{E}}_{v}([\mathbf{X}])$,

$\forall y \in \underline{\mathrm{E}}_{v}([\mathbf{X}]), \exists X \in[\mathbf{X}]$ such that $y \in \underline{\mathrm{E}}_{v}(X)$.

Proof: The first inclusion of the Proposition can be easily proven by considering the monotony of the Choquet integral associated tothe fact that $X \in[\mathbf{X}]$ entails $\underline{X} \leq X \leq \bar{X}$. 
The proof of the second inclusion is less staightforward. Let $y$ be an element of the interval $\underline{\mathrm{E}}_{v}([\mathbf{X}])$. Let $X$ be a vector of $[\mathbf{X}]$. If $y \in \underline{\mathrm{E}}_{v}(X)$ then the proof is ended. If not, let $z=\min (\mid y-$ $\left.\check{C}_{v}(X)|| y-,\check{C}_{v^{c}}(X) \mid\right)$. Let $Z$ be the vector whose elements are equal to $z$. By construction, either $y \in \underline{\mathrm{E}}_{v}(X+Z)$ or $y \in \underline{\mathrm{E}}_{v}(X-Z)$. Since the Choquet integral is an increasing function, by construction $(X+Z) \in[\mathbf{X}]$ and $(X-Z) \in[\mathbf{X}]$, which ends the proof.

For the third step, it seems natural to define the interval-valued expectation operators for intervalvalued vectors as follows:

\section{Definition 4}

$\underline{\overline{\mathrm{E}}}_{v}([\mathbf{X}])=\left[\check{C}_{v^{c}}(\underline{X}), \check{C}_{v}(\bar{X})\right]$

$\underline{\underline{\underline{B}}}_{v}([\mathbf{X}])=\left[\min \left(\check{S}_{v^{c}}(\underline{X}), \check{S}_{v}(\bar{X})\right), \max \left(\check{S}_{v^{c}}(\underline{X}), \check{S}_{v}(\bar{X})\right)\right]$.

The Property ?? of inclusion still holds for interval-valued vectors.

\section{Proposition 8}

$$
\underline{\underline{\underline{B}}}_{v}([\mathbf{X}]) \subseteq \overline{\mathrm{E}}_{v}([\mathbf{X}]) .
$$

Proof: It is sufficient to prove that $\check{S}_{v^{c}}(\underline{X})$ and $\check{S}_{v}(\bar{X})$ belong to $\left[\check{C}_{v^{c}}(\underline{X}), \check{C}_{v}(\bar{X})\right]$. Considering Proposition ?? and the fact that the Choquet integral is monotonic with respect to its integrand, we can write the following inequalities:

- $\check{S}_{v^{c}}(\underline{X}) \leq \check{C}_{v}(\underline{X}) \leq \check{C}_{v}(\bar{X})$ and $\breve{S}_{v^{c}}(\underline{X}) \geq C_{v^{c}}(\underline{X})$ which implies $\check{S}_{v^{c}}(\underline{X}) \in\left[\check{C}_{v^{c}}(\underline{X}), \check{C}_{v}(\bar{X})\right]$.

- $\check{S}_{v}(\bar{X}) \leq \check{C}_{v}(\bar{X})$ and $\check{S}_{v}(\bar{X}) \geq$ $\breve{C}_{v^{c}}(\bar{X}) \geq \breve{C}_{v^{c}}(\underline{X})$ which implies $\check{S}_{v}(\bar{X}) \in\left[\check{C}_{v^{c}}(\underline{X}), \check{C}_{v}(\bar{X})\right]$.

Note that the fact that $\underline{\underline{G}}_{v}([\mathbf{X}])$ is symmetrically distributed in $\underline{\mathrm{E}}_{v}([\mathbf{X}])$ (Proposition ??) does not hold for an interval-valued input. However, the decomposition principle presented in [?] and Proposition ?? still holds.

\section{Proposition 9}

$$
\begin{aligned}
& \overline{\mathrm{E}}_{v}([\mathbf{X}])=\overline{\mathrm{E}}_{v}\left(\left[\mathbf{X}^{+}\right]\right) \ominus \overline{\overline{\mathrm{E}}}_{v}\left(\left[\mathbf{X}^{-}\right]\right) \\
& \underline{\overline{\mathrm{B}}}_{v}([\mathbf{X}])=\underline{\overline{\mathrm{E}}}_{v}\left(\left[\mathbf{X}^{+}\right]\right) \boxminus \underline{\overline{\mathrm{E}}}_{v}\left(\left[\mathbf{X}^{-}\right]\right)
\end{aligned}
$$

Proof: Let $[\mathbf{X}]$ be an interval-valued vector and $\left[\mathbf{X}^{+}\right]$and $\left[\mathbf{X}^{-}\right]$the decomposition proposed in Proposition ??: $[\mathbf{X}]=\left[\mathbf{X}^{+}\right] \ominus\left[\mathbf{X}^{-}\right]$i.e. $\underline{X}=$ $\underline{X}^{+}-\bar{X}^{-}$and $\bar{X}=\bar{X}^{+}-\underline{X}^{-}$. Therefore,

$\underline{\mathrm{E}}_{v}([X])=\left[\check{C}_{v^{c}}(\underline{X}), \underline{C_{v}}(\bar{X})\right]=\left[C_{v^{c}}\left(\underline{X}^{+}\right)-\right.$ $\left.C_{v}\left(\bar{X}^{-}\right), C_{v}\left(\bar{X}^{+}\right)-C_{v^{c}}\left(\underline{X}^{-}\right)\right]$.

Considering

$$
\begin{aligned}
& \underline{\overline{\mathrm{E}}}_{v}\left(\left[\mathbf{X}^{+}\right]\right)=\left[C_{v^{c}}\left(\underline{X}^{+}\right), C_{v}\left(\bar{X}^{+}\right)\right] \text {and } \\
& \overline{\overline{\mathrm{E}}}_{v}\left(\left[\mathbf{X}^{-}\right]\right)=\left[C_{v^{c}}\left(\underline{X}^{-}\right), C_{v}(\bar{X})\right],
\end{aligned}
$$

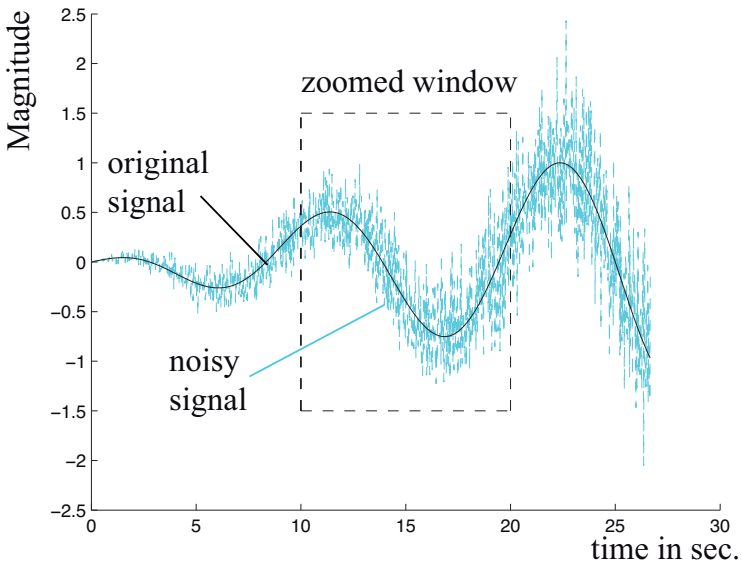

Figure 1: Original signal (black) superimposed on the noisy signal (cyan).

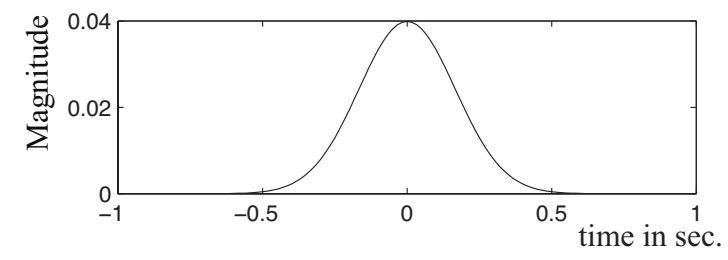

Figure 2: Gaussian filter.

and the definition of the Minkowski subtraction we obtain $\overline{\mathrm{E}}_{v}\left(\left[X^{+}\right]\right) \ominus \underline{\mathrm{E}}_{v}\left(\left[X^{-}\right]\right)=$ $\left[C_{v^{c}}\left(\underline{X}^{+}\right)-C_{v}\left(\bar{X}^{-}\right), C_{v}(\bar{X}+)-C_{v^{c}}\left(\underline{X}^{-}\right)\right]$.

The proof of the second equality is similar when considering the dual Minkowski subtraction.

\section{Experiments}

This section aims at illustrating the different properties of the imprecise-valued filtering method we propose. This experiment is based on considering a synthetic signal of the form $x(t)=k t \cos (\omega t)$ degraded by additive gaussian noise whose standard deviation increases with time (i.e. the noise is not stationary) as depicted in Figure ??.

The filter we use is a low-pass Gaussian filter with a standard deviation equal to 10 times the sampling period (see Figure ??). In this experiment, we suppose the filter to be correctly calibrated to filter the additive noise - even if this hypothesis is not true since the noise is not stationary.

On one hand, we achieve a precise filtering of the discrete signal by using a standard (Dirac) sampling of the Gaussian impulse response. On the other hand, we achieve an imprecise filtering by using a discrete capacity designed to account for the fact that the sampling kernel is unknown. The core of the capacity contains all the discrete impulse responses that would have been obtained by sampling the Gaussian impulse response with a sampling kernel that respects the Shannon sampling theorem i.e. 


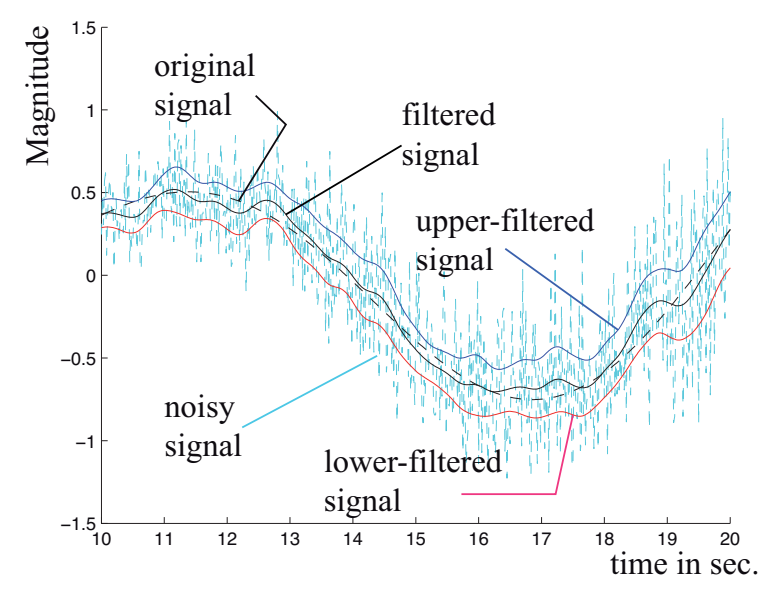

Figure 3: Precise signal filtered with the asymmetric Choquet integral based approach.

whose bandwidth is less than half the sampling period (see [?]).

In the first part of the experiment, we consider filtering the above defined noisy precise signal by using both the asymmetric and the symmetric Choquet based approaches. Figures ?? and ?? show a zoomed window (see Figure ??) of the result of this experiment. On both Figures, the original signal is plotted in dotted black, the noisy signal is plotted in cyan, the precise output of the precise filter is plotted in black and the imprecise output of the capacity-based filter is plotted in red (lower value) and blue (upper value). For both approaches, the output of the precise filter (plain black line) is always included in the output of the imprecise filter (since the capacity dominates the probability measure induced by the discrete impulse response). However, the original signal does not fully belongs to the interval-valued filtered signals. In fact, since the continuous Gaussian filter is not appropriate for filtering the whole continuous signal, none of the discrete filters represented by the considered capacity is appropriate to filter the discrete signal. As can be easily remarked, by comparing Figure ?? with Figure ??, the Šipoš -based approach leads to a more specific signal when the signal value is near 0 compared to the Choquet-based approach. This comparison is more straightforward by looking on Figure ??. In fact, in the Šipoš -based approach, the value 0 has a special role of breaking point. This special role is particularly relevant for designing feedback filters in automatic control processes since a maximal specificity of the output value of the filter is required when the process is close to the nominal equilibrium point.

In the second part of the experiment, the input signal is imprecise. More precisely, we suppose we know the fact that the standard deviation of the additive noise increases with time. Within the classical approach, this knowledge would have been used by empirically modifying the bandwidth of the

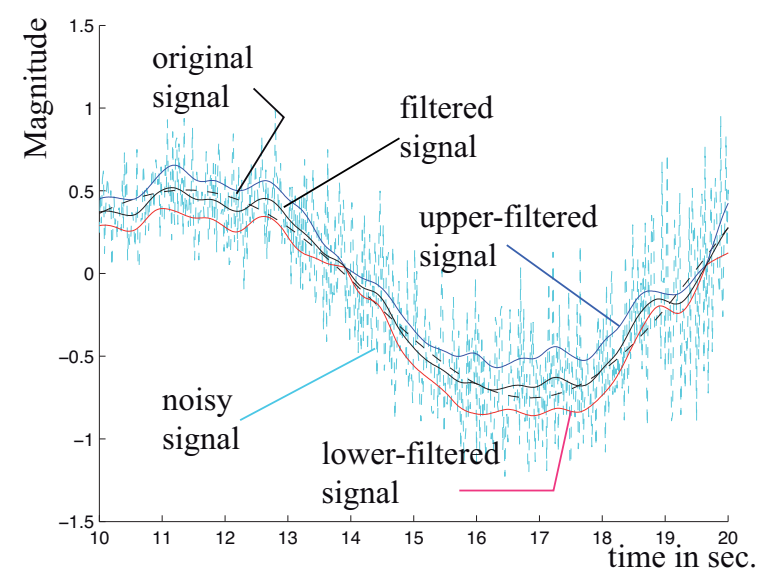

Figure 4: Precise signal filtered with the symmetric Choquet (Sipos) integral based approach.

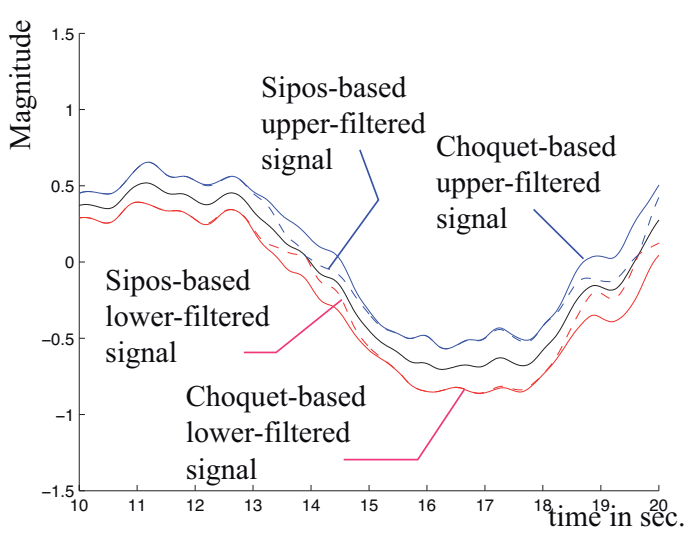

Figure 5: Comparison of the signal filtered with the asymmetric Choquet integral (blue-upper, redlower) and the symmetric Choquet integral (dotted blue-upper, dotted red-lower) .

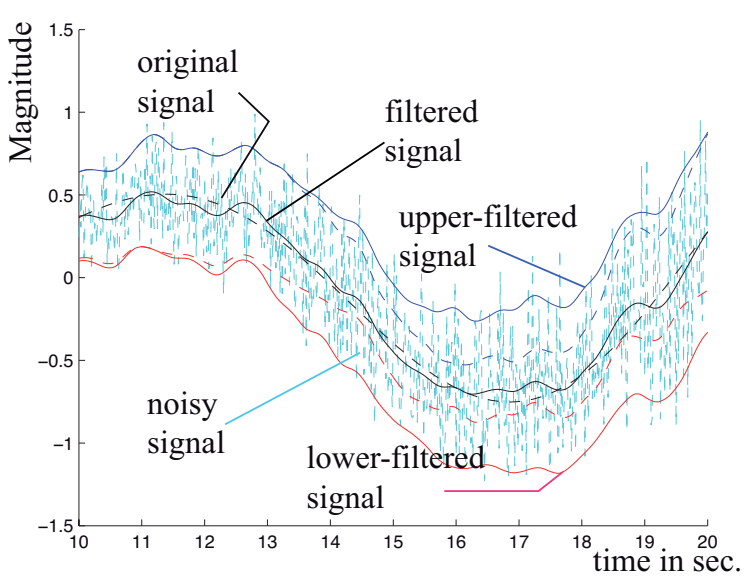

Figure 6: Imprecise signal filtered with the asymetric Choquet integral based approach. 


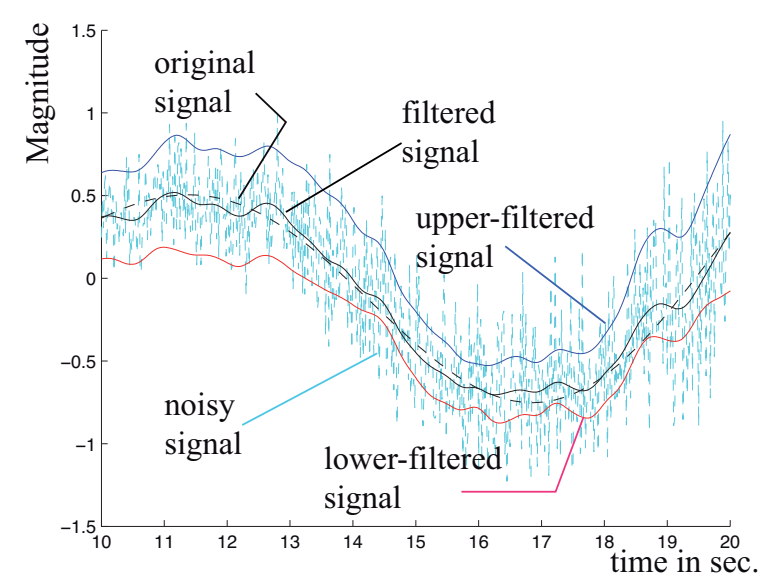

Figure 7: Imprecise signal filtered with the symetric Choquet (Sipos) integral based approach.

Gaussian filter. Within our approach, we use this knowledge to construct, for each sample of the signal to be filtered, a $60 \%$ confidence interval. We thus construct an interval-valued noisy signal that should contain $60 \%$ of the real values of the original signal. Figures ?? and ?? show a zoomed window of the result of this experiment. The color coding used in Figures ?? and ?? is the same than in Figures ?? and ??. The Šipoš -based filtered signal is included in the Choquet-based filtered signal (the Šipoš -based filtered signal is represented in dotted lines in Figure ??). All the remarks done in the first part of the experiment (with the precise signal) still hold with the second part of the experiment, except that, since the noise is accounted by the intervalvalued input, the original signal fully belongs to the interval-valued outputs, for both approaches.

\section{Conclusion}

In this paper, we have proposed two extensions of the imprecise-valued filtering method we have previously proposed in [?]. This method allows representing a partial lack of knowledge about the impulse response of the filter to be used. It consists in replacing the classical single precise impulse response by a set of impulse responses that is consistent with the user's expert knowledge. The set of impulse responses is represented by a concave capacity and the aggregation operator used in linear filtering is replaced by a Choquet integral. Due to these replacements, the computational complexity of this new approach is comparable to the complexity of the classical approach. The first extension concerns the use of the Šipoš integral. This extension leads to a filtering approach that achieve a kind of compensation between positive and negative part of the signal. The obtained signal is thus more specific near a nominal equilibrium point than the previous approach proposed in [?]. The second extension allows the use of the imprecise filtering approach with imprecise inputs. In fact, the input of a filter can be imprecise either because this signal is the output of another imprecise filter (and thus it allows iterative filtering methods), or because this imprecision accounts for a known (calibrated) imprecision or for imprecision due to quantification.

\section{References}

[1] J. Serra, Academic Press Inc. Image analysis and mathematical morphology, London, 1982.

[2] Denneberg, Kluwer Academic Publishers. NonAdditive Measure and Integral, 1994.

[3] K. Loquin and O. Strauss, On the granularity of summative kernels Fuzzy Sets and Systems, 159: 1952-1972, 2008.

[4] A. Rico and O. Strauss, Imprecise expectations for imprecise linear filtering, International Journal of Approximative Reasoning, 51: 933-947, 2010

[5] R. Yager and V. Kreinovich, Decision making under interval probabilities. International Journal of Approximate Reasoning, 22: 195-215, 1999.

[6] M. Hukuhara, Intégration des applications mesurables dont la valeur est un compact convexe, Funkcialaj Ekvacioj, 10: 205-223, 1967.

[7] K. Loquin, O. Strauss and Jean-Francois Crouzet Possibilistic signal processing: How to handle noise? International Journal of Approximative Reasoning 51: 1129-1144, 2010. 